\title{
On the fault location algorithm for distribution networks in presence of DG
}

\begin{abstract}
Connecting distributed generation (DG) units to the distribute networks impose several impacts on it which have not been considered in conventional fault location algorithms. This paper presents an accurate fault location technique for unbalanced radial distribution networks based on evaluating measured values of short Circuit Current (S/C.C) at the source bus with a designed Multi-Layer Feed Forwarded Neural Network (ML-FFNN). The estimated locations of different fault types are compared with the actual distances and Average Difference Percentage (ADP) is calculated for each fault type. The designed neural network is able to work with small scale datasets. Hence the proposed method can be implemented in the real distribution networks.
\end{abstract}

Keyword: Fault location; Distribution network; Distribution generation (DG); Short circuit current 